\title{
CREACIÓN CONSTITUCIONAL EN SOCIEDADES PLURALES. LA "ESTRATEGIA DE ACUMULACIÓN"
}

Roberto Gargarella Mariotti 


\section{ROBERTO GARGARELLA MARIOT'TI}

Sociólogo, abogado y Doctor en Derecho por la Universidad de Buenos Aires, L.L.M. por la Escuela de Leyes de la Universidad de Chicago, Master en Ciencia Política por la Facultad Latinoamericana de Ciencias Sociales, J.S.D. por la Universidad de Chicago, y Post-Doctorado en el Balliol College, Oxford. Es Profesor Visitante de la Escuela de Derecho de la Universidad Torcuato Di Tella y Profesor Titular de la Facultad de Derecho de la Universidad de Buenos Aires. Ha sido académico e investigador visitante en varias universidades extranjeras, además de ser autor de numerosos libros y artículos, entre ellos "The Legal Foundations of Inequality" (Cambridge University Press, 2010) y "Latin American Constitutionalism" (Oxford University Press, 2013). 


\section{CREACIÓN CONSTITUCIONAL EN SOCIEDADES PLURALES. LA "ESTRATEGIA DE ACUMULACIÓN"}

\section{INTRODUCCIÓN}

La pregunta que quiero explorar en este artículo es cómo manejar situaciones de marcado pluralismo, como aquellas que normalmente caracterizan los procesos contemporáneos de elaboración de constituciones. En lo sucesivo, describiré y examinaré críticamente tales situaciones. Me referiré a cuatro respuestas distintas para el problema del pluralismo, que pueden encontrarse en la historia de las convenciones constitucionales. Denominaré a estas respuestas: imposición, silencio, sintesis y acumulación, y las ilustraré con ejemplos que provienen de la historia constitucional de América Latina. Mi sugerencia es que en situaciones que están lejos de ser ideales, es decir, situaciones que no se relacionan con lo que la doctrina ha llamado "momentos constitucionales", los demócratas deberían deliberar para alcanzar acuerdos "sintéticos" —en otras palabras, debieran empeñarse para encontrar puntos comunes de acuerdo con las propuestas más razonables ofrecidas por sus oponentes.

i) Imposición. La primera respuesta que exploraré, la imposición, era frecuente en países social y culturalmente heterogéneos controlados por una determinada facción. La imposición implica que uno de los grupos involucrados consigue imponer su propia voluntad, desplazando las demandas del resto. La respuesta basada en la imposición legal fue la más común entre los conservadores latinoamericanos durante el siglo XIX. Durante ese periodo, y particularmente durante su primera mitad, los grupos conservadores obtuvieron el control sobre la política y, por consiguiente, promulgaron las constituciones favorecidas por ellos, reflejando inequívocamente su principal preocupación: la necesidad de evitar el deterioro de la moral que percibían en sus respectivos países. Frecuentemente, estas constituciones incluyeron una religión oficial, promovieron una visión moral comprensiva y definieron una lista de derechos que era interpretada de modo consistente con (y dependiente de) el proyecto moral dominante. En algunos casos — los más extremos e infrecuentestales constituciones establecieron un sistema de premios y castigos para aquellos 
que mostraban conductas que eran rechazadas o favorecidas por los funcionarios en el poder ${ }^{1}$.

ii) Silencio. La segunda respuesta, el silencio, puede aparecer cuando las partes no encuentran la manera de resolver sus desacuerdos. En estas situaciones, los partícipes deciden dejar irresuelta la cuestión sobre la que no llegan a definir una respuesta compartida. Como sostuviera el constitucionalista Cass Sunstein, la idea es "dejar la cuestión abierta" (leaving things undecided, Sunstein 1999, 3). Existe un ejemplo interesante de este tipo de respuesta en los debates constitucionales mexicanos de 1857. Durante esta convención, una de las disputas de mayor duración e intensidad se refirió al lugar que debía ocupar la religión y la tolerancia religiosa en el nuevo ordenamiento. De cara a esta compleja discusión, los delegados optaron por la indecisión. En otros términos, dejaron la cuestión irresuelta. El tema religioso, en el México de la época, representaba un problema particularmente apremiante en razón de los enormes privilegios de que gozaba la Iglesia en ese momento, lo cual llevó a muchos liberales a oponerse a la iniciativa que pretendía ratificar las injustas

1. Uno de los ejemplos más extremos en este sentido fue la Constitución chilena de 1823, redactada por el jurista conservador Juan Egaña. Egaña y su Constitución fueron enormemente influyentes en Chile y, más generalmente, en la región, pese a que fue de corta duración. La Carta Magna de Egaña contemplaba un Ejecutivo fuerte, el cual, en opinión de Egaña, controlaba "la totalidad de la administración, sin interferencia de la legislatura, la cual debía promulgar sólo algunas leyes generales y permanentes, y quienes se reunirían sólo después de largos intervalos y por un tiempo muy breve" (Silva Castro 1969, 86-7). Una de las principales funciones del Presidente era asegurar el respeto de la religión católica, la cual se estableció como la religión oficial del país. Además, con el objeto de asegurar la imposición de la religión oficial, la Constitución creó un "Senado conservador" a cargo de controlar la "moralidad y hábitos nacionales" y, más radicalmente, acompañó el texto con un "Código Moral” sustantivo, cuyo objeto era regular la vida moral de los habitantes chilenos hasta en los detalles más pequeños. En opinión de Egaña, el "Código Moral” representaba la expresión más alta y meditada de su vida de reflexiones teóricas sobre la moralidad. La primera parte del Código se refería a la religión y a la necesidad de protegerla (regulaba, por ejemplo, la forma en que debían celebrarse las festividades públicas de la Iglesia, como también las relaciones entre las personas y sus confesores). En su segunda parte, el Código analizaba la familia, su composición y relaciones entre sus miembros (se refería a las actitudes personales y comportamiento, incluyendo la ingratitud, vanidad, denigración o el abandono de los padres). Su tercera parte se relacionada con la educación, la cual tenía un rol fundamental en el proyecto de Egaña. El Código reguló el consumo de alcohol, estableció estrictos parámetros a seguir en el desarrollo de ceremonias públicas y privadas, y estableció la prohibición de repartir panfletos sin la autorización previa de un grupo de censuradores. También incluyó sanciones estrictas para los ciudadanos que "crearan partidos políticos y expusieran sus opiniones francamente, o aquellos que se reunieran en lugares públicos" (ibid., 637-8). Por extremo que fuera, el Código fue muy influyente entre los conservadores latinoamericanos. 
ventajas adquiridas por la Iglesia a lo largo de muchos años ${ }^{2}$. Su poder de influencia, sin embargo, era limitado en relación con el peso alcanzado por los sostenedores de los privilegios eclesiásticos. Lo que los delegados decidieron hacer, en definitiva — dejando de manifiesto el carácter transaccional de la Constitución—, fue mantener silencio con respecto a la cuestión religiosa, impidiendo, al menos, que la Constitución se convirtiera en un documento intolerante en este sentido. De este modo, lograron al menos impedir el establecimiento de la intolerancia religiosa.

iii) Sintesis. La tercera respuesta, de sintesis, es la que creo deberían favorecer los demócratas deliberativos. Podemos relacionar esta respuesta con la noción rawlsiana del consenso superpuesto (overlapping consensus), conforme a la cual distintos grupos apoyan una solución común por distintos motivos (propios de sus respectivas visiones). Para alcanzar un acuerdo sintético —un acuerdo que todos podemos compartir- puede ser necesario que cada parte despliegue un esfuerzo significativo: cada parte debe dejar de lado o dejar en suspenso determinados aspectos relevantes de sus propias propuestas. Encontramos un interesante ejemplo de esta respuesta en los debates iniciales de la Constitución de los Estados Unidos con respecto a la religión. Este tópico fue una de las materias más divisorias entre los distintos grupos durante el "periodo fundacional". Con anterioridad a la Constitución, la situación imperante parecía ser de imposición severa: existía un establecimiento religioso en Nueva Inglaterra con la Iglesia Congregacional, y en el sur con la Iglesia Anglicana. Distintas sectas que habían sufrido persecución religiosa en Inglaterra ahora ejercían presión para el avance de sus propias posiciones a través del uso de los poderes coercitivos del Estado. Al final, sin embargo, la mayoría de los grupos sociales aceptó una cláusula de no establecimiento (aquello fue primeramente aceptado en Virginia y luego incorporado en la Constitución) ${ }^{3}$ debido a diversas razones, incluyendo auto-protección, reciprocidad, tolerancia, secularismo, etc. Por

2. Por ejemplo, el delegado Zarco, una de las figuras más importantes de la convención, rechazó el establecimiento de la religión católica señalando -al contrario de sus creencias personalesque el rol asumido por la Iglesia mexicana durante todos esos años era inaceptable. "Se ha desnaturalizado la religión de Cristo porque se ha declarado como enemiga de la liberad; ha acumulado riqueza empobreciendo al país; ha engañado a la gente... ha defendido privilegios y dinero, ignorando las verdades del catolicismo". (Zevada 1972, 38-9). Sin embargo, al final, los liberales no fueron capaces de garantizar la tolerancia religiosa a través de la Constitución, dadas las diferencias presentes no sólo respecto de los representantes conservadores, sino incluso dentro del grupo liberal.

3. La Primera Enmienda de la Constitución indica lo siguiente: "El Congreso no dictará ley alguna con respecto al establecimiento de la religión, ni prohibirá su libre ejercicio, ni coartará la libertad de prensa, ni el derecho del pueblo a reunirse pacíficamente o a pedir al Gobierno remedio de agravios". 
lo tanto, no es sorprendente que el caso de la Primera Enmienda represente uno de los principales ejemplos de Rawls para ilustrar sus reflexiones sobre la razón pública, coerción estatal y consenso superpuesto ${ }^{4}$.

iv) Acumulación. La respuesta final, acumulación, aparece cuando los distintos participantes, por algún motivo, encuentran muy difícil alcanzar un acuerdo común $y$, al mismo tiempo, rechazan permanecer en silencio con respecto al tema en cuestión (ver Lerner 2013, 2016). En aquellos casos, los miembros de la convención pueden decidir simplemente sumar o juntar (unas encimas de las otras) sus distintas pretensiones, dejando sus demandas en tensión -total o casi totalmente intactas. Dado que esta estrategia se volvió, en mi opinión, la más importante, distintiva e influyente en el constitucionalismo de Latinoamérica (es decir, en la región en la cual enfoco mi análisis), voy a prestar particular atención a esta elección específica. Para tales efectos, presentaré distintos ejemplos de preferencia por esta estrategia en Latinoamérica, desde su independencia hasta el día de hoy.

\section{“ESTRATEGIA DE ACUMULACIÓN” DE LATINOAMÉRICA}

En el siglo XIX, y con posterioridad al periodo de independencia, la "estrategia de acumulación" se volvió particularmente relevante en Latinoamérica. La preferencia por la "acumulación" se tornó evidente en las dos principales áreas de la Constitución, es decir, aquella relacionada con la organización del poder, a la que podemos llamar parte orgánica; y aquella relacionada con la declaración de derechos, a la que denominamos parte dogmática. Dadas las dificultades que encontraron al negociar sus diferencias y resumir sus pretensiones frecuentemente opuestas, muy habitualmente conservadores y liberales decidieron sumar dichas pretensiones contradictorias en el mismo texto.

Con respecto a la organización de poderes, los liberales propusieron un sistema de frenos y contrapesos (como aquél que había sido adoptado en los Estados Unidos), mientras los conservadores prefirieron la creación de un Ejecutivo completamente poderoso (como aquél que había sido establecido, por ejemplo, en la influyente, estable y autoritaria Constitución chilena de 1833). Ahora bien, debido a las dificultades que encontraron en la negociación de sus diferencias al respecto, liberales y conservadores, en la mayoría de los países latinoamericanos, decidieron redactar constituciones que incluyeran ambos tipos de pretensiones opuestas. De este modo, crearon cartas magnas que definían la creación de un sistema de "frenos

4. En contrario a esta opinión, Lerner $(2013,2016)$. 
y contrapesos" (permitiendo a cada rama del poder controlar a las otras), y al mismo tiempo establecieron un Ejecutivo súper poderoso (dando lugar a los llamados sistemas híper-presidencialistas, Nino 1996). Esta última opción quedó reflejada a través del hecho de que, de acuerdo a las demandas propias de los modelos conservadores de constitucionalismo, consagraron prerrogativas especiales para el Presidente (por ejemplo, con respecto a la declaración de estado de sitio o la intervención en los asuntos de estados locales), que quedaron superpuestas a un esquema general de "frenos y contrapesos", como el que proponía el liberalismo. La decisión que primó fue entonces la de "acumular" ambas propuestas opuestas en el mismo texto. Esta combinación peculiar — podríamos decir- se convertiría desde entonces en la característica principal y más distintiva del constitucionalismo latinoamericano hasta nuestros tiempos.

Lo que las constituciones latinoamericanas hicieron con respecto a la organización de poderes se hizo evidente, asimismo, con respecto a lo que hicieron en relación con las declaraciones de derechos. Un buen ejemplo de esto se encuentra en la influyente Constitución argentina de 1853. En esa época, en Argentina, como en varios otros países latinoamericanos, los grupos liberales y conservadores se enfrentaron de forma airada sobre variados temas, y de manera particular en torno a la cuestión religiosa. Específicamente, los liberales favorecían la tolerancia religiosa, mientras que los conservadores proponían la adopción de la religión católica como culto oficial. Frente a dichos conflictos, los delegados de la Constitución argentina de 1853, que incluían representantes de los grupos liberales y conservadores, decidieron primeramente, y siguiendo las demandas de los conservadores, otorgar un estatus especial a la Iglesia Católica a través del artículo 2 de la Constitución ("El Gobierno federal sostiene el culto Católico Apostólico Romano"). Al mismo tiempo, pero siguiendo ahora las demandas de los liberales, adoptaron un esquema de tolerancia religiosa a través del artículo 14 de la Constitución ("Todos los habitantes de la Confederación gozan de los siguientes derechos... de profesar libremente su culto"). Es decir, incluyeron en la Constitución ambos compromisos contradictorios al mismo tiempo.

Encontramos exactamente la misma tendencia de "acumulación" en uno de los artículos más interesantes de la Constitución argentina de 1853, el artículo 19, el cual se refiere a temas de moralidad privada. En su formulación inicial, la cual apareció en los primeros documentos constitucionales y en el primer borrador del documento de 1853, Argentina adhirió a una fórmula típicamente liberal al respecto. De acuerdo con ella, el Estado aseguraría la protección de la moralidad privada, "las acciones privadas de los hombres", en la medida en que dichas acciones no dañasen a terceros. Esta formulación inicial representó lo que yo llamaría el "sueño de John Stuart Mill" y fue apoyada en mayor o menor medida por un gran número de 
liberales. Sin embargo, durante los debates constitucionales, los representantes de los grupos conservadores - liderados por el convencional Ferré, de Corrientesse opusieron a la adopción de la declaración anterior y a otras cláusulas liberales de la Constitución, y sostuvieron que no firmarían dicho documento liberal. A partir de dicha amenaza lograron introducir distintas reformas en diferentes aspectos del borrador, incluyendo la formulación inicial del artículo 19. Esto dañó profundamente la formulación inicial de dicho artículo, arruinando el artículo soñado por John Stuart Mill. Básicamente, los conservadores limitaron dicho compromiso liberal (el cual otorgaba protección estricta a los temas de moralidad privada) a través de las ideas de "orden y moralidad pública". Por lo tanto, de acuerdo con la última formulación del artículo 19 (la cual continúa vigente), las acciones privadas están protegidas por el Estado en la medida en que no afecten el "orden público y la moral" (la cual no era la "pesadilla" a la cual se refería John Stuart Mill, pero se le acercaba bastante).

Si bien las soluciones implementadas por la Constitución argentina de 1853 fueron, en este sentido, bastante incómodas, el hecho es que representan - y siguen representando - la principal estrategia constitucional adoptada en América Latina en situaciones de profundo pluralismo social.

Permítanme concluir la presente sección sugiriendo que la "estrategia de acumulación", la que adquirió tanta influencia en la América Latina del siglo XIX, no fue abandonada en las décadas siguientes. Más bien al contrario. Según entiendo, dicha estrategia continúa siendo una característica crucial y distintiva del constitucionalismo latinoamericano contemporáneo. Por ejemplo, la Constitución de Colombia de 1991 o la Constitución peruana actual — para citar dos textos de referencia permanente en la región - nos muestran cartas fundamentales que combinan (lo que podría llamarse) compromisos económicos "socialistas" y "neoliberales". De hecho, ambas incluyen fuertes cláusulas sociales, a la vez que establecen fuertes protecciones al derecho de propiedad, los mercados e inversiones privadas.

De diferentes maneras seguimos encontrando el mismo tipo de “acumulación' en la mayoría de las constituciones latinoamericanas contemporáneas. La vasta mayoría de ellas combinan declaraciones de derechos modernas al estilo del siglo XXI (las cuales incluyen derechos de participación y referencias a los derechos de minorías sexuales, religiosas, étnicas, raciales o nacionales) con una organización de poderes anticuada, al estilo del siglo XVIII, la que se basaba en un entendimiento restrictivo o elitista de la democracia. Desde mi punto de vista, esta combinación representa el aspecto más preocupante del constitucionalismo contemporáneo latinoamericano, conforme con lo que discutiremos a continuación (Gargarella 2010, 2013). 
EL FRACASO DE LA "ESTRATEGIA DE ACUMULACIÓN". EL EJEMPLO LATINOAMERICANO Y "LA SALA DE MÁQUINAS” DE LA CONSTITUCIÓN.

La idea de "mezclar" posturas opuestas, superponiendo ciertas pretensiones con otras en tensión con ellas, aparece arraigada en la tradición constitucional de Latinoamérica. Después de todo, fue el mecanismo primario para llegar a acuerdos entre liberales y conservadores durante el siglo XIX. Como hemos comentado, era práctica común superponer aspiraciones esencialmente liberales con otras conservadoras. La "acumulación” de demandas que colisionaban entre sí representa una manera aparente de introducir tensiones e inconsistencias dentro de la estructura constitucional.

Las tensiones y contradicciones internas existentes en una Constitución pueden ser de distinto tipo. Algunas de estas tensiones pueden ser internas respecto de una sección de la Constitución - llamémoslas tensiones intra-seccionales (es decir, tensiones internas a aquella parte de la Constitución dedicada a la declaración de derechos; o internas a la organización del gobierno). También pueden surgir tensiones entre distintas partes de la Constitución — llamemos a éstas tensiones inter-seccionales (es decir, derechos vs. poder).

Por ejemplo, la introducción de referencias a la "propiedad comunal" en constituciones recientes, tales como las de Ecuador y Bolivia, conlleva problemas de operativización más o menos obvios. Podemos apreciar la intención detrás de la incorporación de "principios interpretativos" que son distintos a los tradicionales (ver más abajo). Sin embargo, es difícil no preguntarse cómo es que estos principios han de ser entendidos cuando la Constitución no repudia otros principios e instituciones que son contradictorios en relación con ellos - típicamente, aquellos asociados con derechos de propiedad tradicionales (liberales, "clásicos"). Ésta parece ser una buena ilustración de lo que identifiqué como tensión intra-seccional.

El problema generado por esta "estrategia de acumulación" se torna aún más claro en lo que se relaciona con la organización de poderes. En prácticamente todos los países latinoamericanos, el esquema liberal de "frenos y contrapesos" resulta combinado — sin remordimientos - con un Poder Ejecutivo abrumador. Esta combinación probó ser fatal desde un comienzo. De hecho, la idea de tener una organización de poderes híper-presidencialista conspira directamente contra el éxito del sistema de "frenos y contrapesos", socava la lógica misma del sistema 5 .

5. De hecho, la única razón para tener un sistema de "pesos y contrapesos" está en prevenir las agresiones mutuas entre las ramas y así evitar una "concentración gradual” de los poderes 
Parece claro que si una de las ramas recibe muchos más poderes que las otras, entonces el propósito mismo del sistema de contrapesos mutuos (evitando la "concentración gradual de varios poderes en un mismo departamento") se disuelve. De esta manera, la estructura entera de controles mutuos pasa a convertirse en una virtualmente inútil: lo que la Constitución otorga con una mano - un sistema de equilibrios y controles mutuos- lo quita con la otra, estableciendo en un mismo acto una rama que es de forma sustancial más poderosa que las demás.

El caso que ha representado, probablemente, el fracaso más importante y grave de la "estrategia de acumulación" en América Latina es el que se refiere a las tensiones inter-seccionales. El ejemplo que mejor ilustra estas tensiones es uno que afecta a la vasta mayoría de las constituciones latinoamericanas, específicamente la decisión de establecer organizaciones de poderes híper-presidencialistas junto con declaraciones progresivas de derechos que incluyen, típicamente, numerosos nuevos derechos participativos (Gargarella 2010; Gargarella 2013). En efecto, la notoria "doble marca" del constitucionalismo regional — ramas de gobierno organizadas conforme con reglas que prevalecían en el siglo XIX; derechos establecidos siguiendo pautas muy de avanzadas, propias del siglo XX o XXI- revela un inusual compromiso democrático de dos caras. Como resultado, la estructura de poder correspondía, y sigue correspondiendo, a principios democráticos del siglo XIX: baja participación popular, exclusión de sectores enteros, derechos políticos limitados; los mecanismos emblemáticos de la democracia elitista. Mientras tanto, las nuevas declaraciones de derechos parecen estar vinculadas con una "nueva generación" de discursos y principios democráticos. Éstos apuntan a una amplia participación popular, cuyo apoyo es buscado de distintas maneras; oportunidades institucionales que se han abierto al público para un mayor poder de control y decisión (estableciendo consultas populares, etc.); derechos políticos expandidos; y, simultáneamente, compromisos efectuados con respecto a derechos sociales que buscan promover una mayor participación política de las mayorías (todos los cuales, debo insistir, están sujetos a variadas limitaciones).

Existe un problema obvio - en mi opinión — cuando la meta es promover la participación popular y se organiza, sin embargo, el poder de un modo como el

en mano de una sola de ellas. Tal como comentó James Madison en los Federalist Papers n. 51, "la gran seguridad en contra de la concentración gradual de varios poderes en un mismo departamento, consiste en otorgar a aquellos que administran cada departamento los medios constitucionales necesarios y motivaciones personales para resistir la injerencia de los demás. La disposición de defensa en este caso, tal como en todos los demás, deberá ser proporcional al peligro de ataque. La ambición debe contrarrestar a la ambición. El interés del hombre debe estar conectado con los derechos constitucionales del lugar". 
descrito. Ello, ya que se debe confiar, para la implementación de los mecanismos participativos, en aquellas personas cuyo poder se verá menoscabado cuando la participación del caso se torne efectiva ${ }^{6}$. En este caso, más que en cualquier otro, la "estrategia de acumulación" demuestra sus deficiencias y nos ayuda a explicar y entender una de las principales fallas del constitucionalismo contemporáneo en América Latina.

\section{CONCLUSIONES}

En este artículo he defendido sobre todo lo siguiente idea: en sociedades definidas por el hecho del pluralismo razonable, las asambleas constituyentes debieran aspirar a lograr (lo que he llamado) acuerdos "sintéticos" que resuman las demandas opuestas o contradictorias de los distintos grupos. Esta opción, según lo aquí propuesto, sería más coherente con la idea de contar con una sociedad dialogante e inclusiva. Esta idea nos ayuda a desafiar, por lo demás, lo que he llamado la "estrategia de acumulación" que parece prevalecer en el constitucionalismo latinoamericano contemporáneo. La "estrategia de acumulación," que se manifiesta de formas diversas en los ordenamientos constitucionales de la región, representa una de las fallas más graves del constitucionalismo regional, y explica muchos de los problemas que enfrenta, tanto en materia de organización del poder, como en lo que hace a la implementación de los derechos que generosamente consagra.

6. El problema identificado no se despeja por el argumento de que el gran "enemigo" de la participación política popular es la "concentración del poder económico" (Unger 1987). No es solamente que se requiera una respuesta más amplia al problema, sino que también es cierto que la respuesta, desde su concepción, ignora los (referidos) riesgos de mantener el poder político concentrado (en especial en relación con el antedicho objetivo de diluir el poder político) y, además, ignora las formas en que el poder político concentrado tiende a interactuar con o a directamente favorecer la concentración económica (volveré sobre este punto). 


\section{REFERENCIAS}

Alberdi, J.B. (1981), Bases y puntos de partida para la organización política de la República Argentina, Plus Ultra: Buenos Aires.

Bohman, J. (1996), Public deliberation: Pluralism, complexity, and democracy. Cambridge, MA: MIT Press.

Chesterman, S. (2005), "Imposed Constitutions, Imposed Constitutionalism, and Ownership", Connecticut Law Review, vo. 37: 947.

Cohen, J. (1989), "The economic basis of a deliberative democracy," Social Pbilosopby and Policy 6 (2): 25-50.

Dworkin, R. (1997), “The Arduous Virtue of Fidelity: Originalism, Scalia, Tribe, and Nerve," Fordham Law Review, vol. 65, (1997).

Elkins, Z, Ginsburg, T., Blount, J. (2008), "The Citizen as Founder: Public Participation in Constitutional Approval," Temple Law Review, 81 (2), 361-382.

Elkins, Z., Ginsburg T, Melton J. (2009), The Lifespan of Written Constitution, New York: Cambridge University Press.

Elster, J. (1986), "The Market and the Forum: Three Varieties of Political Theory," in Jon Elster and Aanund Hylland eds., Foundations of Social Choice Theory, Cambridge: Cambridge University Press.

Elster, J., ed. (1998), Deliberative democracy. Cambridge: Cambridge Univ. Press.

Elster, J. (1995), "Forces and Mechanisms in the Constitution-Making Process", Duke University School of Law, vol. 45, n. 1, 364-496.

Elster, J. (2015), “The Political Psychology of Constitution-Making”, Princeton, manuscript on file with the author.

Ely, J. (1980), Democracy and Distrust, Cambridge: Harvard University Press.

Gargarella, R.; Domingo, P.; Roux, T. (2006), Courts and Social Transformation in New Democracies, London: Ashgate.

Gargarella, R. (1998), "Full representation, deliberation, and impartiality", in J. Elster, ed., Deliberative Democracy, Cambridge U. P., 260-80.

Gargarella, R. (2010), The Legal Foundations of Inequality, Cambridge: Cambridge University Press.

Gargarella, R. (2013), Latin American Constitutionalism, Oxford: Oxford University Press. 
Gargarella, R. (2014), "We the People Outside of the Constitution: The Dialogic Model of Constitutionalism and the System of Checks and Balances," Current Legal Problems, 67 (1), 1-47.

Ginsburg, T., Elkins, Z., \& Blount, J. (2009), "Does the Process of ConstitutionMaking Matter?”, Ann. Rev.Law.Soc.Sci.5, 201-23.

Goodin, R. (1989), "Laundering Preferences," in J. Elster\&A.Hylland, Foundations of Social Choice Theory, Cambridge: Cambridge University Press.

Habermas, J. (1996), Between Facts and Norms: Contributions to a Discourse Theory on Law and Democracy, Cambridge: MIT Press.

Halperín Donghi, T. (2007), Historia Contemporánea de América Latina, Alianza: Buenos Aires.

Lerner, H. (2013), Making Constitutions in Deeply Divided Societies, Cambridge: Cambridge University Press.

Lerner, H. (2016), "Constituent Assemblies in Divided Socities".

López Restrepo, Andrés (1994). El cambio de modelo de desarrollo de la economía colombiana. Revista Análisis Político, (21). http://bibliotecavirtual.clacso.org. ar/ar/libros/colombia/assets/own/analisis\%20politico\%2021.pdf.

Moehler, D. (2008), Distrusting Democrats: Outcomes of Participatory Constitution-Making, Ann Arbor: The University of Michigan.

Moehler, D. \& Marchant, E. (undated) "A Multi-Dimensional Model of Participatory Constitution Making and Legitimacy"

Negretto, G. (2015), "Constitution Making in Democratic Constitutional Orders. The Problem of Citizen Participation”, Working Paper CIDE, Mexico.

Nino, C. (1996), The Constitution of Deliberative Democracy, New Haven: Yale University Press.

Paine, T. (1987), Thomas Paine Reader, Oxford: Penguin Books.

Pisarello, G. (2014), Procesos constituyentes. Caminos para la ruptura democrática, Madrid: Trotta.

Rawls, J. (1991), Political Liberalism, New York: Columbia University Press.

Salazar, D. (2015), "My Power in the Constitution: The Perversion of the Rule of Law in Ecuador," SELA, Yale University. https://www.law.yale.edu/sites/ default/files/documents/pdf/SELA15_Salazar_CV_Eng.pdf

Sieyés, E. (1990), Escritos y discursos de la revolución, Madrid: Centro de Estudios Constitucionales. 
Sieyés, J. (2014), Emmanuel Joseph Sieyés. The Essential Political Writings, London: Brill Academic Pub., Lam. Edition.

Silva Castro, R. (1969) Juan Egaña. Antología, Santiago de Chile: Editora Andrés Bello.

Sunstein, C. (1991), "Preferences and Politics," Philosophy \& Public Affairs, Vol. 20, No. 1 (Winter), pp. 3-3.

Sunstein, C. (2001), One Case at a Time, Cambridge: Harvard University Press.

Tushnet, M. (1988), Red, White and Blue, Cambridge: Harvard University Press.

Voigt, S. (2003), "The Consequences of Popular Participation in Constitutional Choice. Towards a Comparative Analysis", in A. van Aaken, C. List, C. Luetgeeds, Deliberation and Decision, Aldershot: Ashgate, 199-229.

Widner, J. (2008) "Constitution Writing in Post-Conflict Settings: An Overview, William \& MaryLaw Review 49 (4), 1513

Zevada, R. (1972), La lucha por la libertad en el congreso constituyente de 1857. Elpensamiento de Ponciano Arriaga, México: Ed. Nuestro Tiempo. 